\title{
A Formula for the Möbius Function of the Permutation Poset Based on a Topological Decomposition
}

\author{
Jason P Smith \\ Department of Computer and Information Sciences \\ University of Strathclyde, Glasgow, UK
}

\begin{abstract}
The poset $\mathcal{P}$ of all permutations ordered by pattern containment is a fundamental object of study in the field of permutation patterns. This poset has a very rich and complex topology and an understanding of its Möbius function has proved particularly elusive, although results have been slowly emerging in the last few years. Using a variety of topological techniques we present a two term formula for the Möbius function of intervals in $\mathcal{P}$. The first term in this formula is, up to sign, the number of so called normal occurrences of one permutation in another. Our definition of normal occurrences is similar to those that have appeared in several variations in the literature on the Möbius function of this and other posets, but simpler than most of them. The second term in the formula is (still) complicated, but we conjecture that it equals zero for a significant proportion of intervals. We present some cases where the second term vanishes and others where it is nonzero. Computing the Möbius function recursively from its definition has exponential complexity, whereas the computation of the first term in our formula is polynomial and the exponential part is isolated to the second term, which seems to often vanish. This is thus the first polynomial time formula for the Möbius function of what appears to be a large proportion of all intervals of $\mathcal{P}$.
\end{abstract}

Résumé. L'ensemble partiellement ordonní (EPO) $\mathcal{P}$ de toutes les permutations, ordonnées par inclusion de motifs, est d'importance fondamentale dans l'étude des motifs de permutations. Cet EPO Possède une topologie très riche et complexe ; comprendre la fonction de Möbius associée s'est avéré particulièrement difficile, même si des résultats sont apparus lentement au cours des dernières années. En utilisant divers outils topologiques nous établissons une formule (contenant deux termes) pour la fonction de Möbius appliquée aux intervalles de $\mathcal{P}$. Le premier terme de cette formule est, à un signe près, le nombre des occurrences dites normales d'une permutation dans une autre. Notre définition des occurrences normales est semblable à celles que l'on trouve à plusieurs endroits dans la littérature existante sur la fonction de Möbius, que ce soit de cet EPO ou d'autres ; mais elle semble plus simple que la plupart de celles-ci. Le second terme de la formule est compliqué, mais nous conjecturons qu'il se réduit à zéro pour bon nombre d'intervalles. Nous présentons des exemples oú le second terme est réduit à zéro, ainsi que des exemples où ce n'est pas le cas. Le calcul de la fonction de Möbius en utilisant la formule de récurrence dans sa définition a une complexité exponentielle, tandis que le calcul du premier terme de notre formule a une complexité polynomiale, et la partie exponentielle est isolée dans le second terme, qui semble souvent réduit à zéro. Nous obtenons ainsi le premier algorithme polynomial pour calculer la fonction de Möbius de ce qui semble être une part importante d'intervalles de $\mathcal{P}$.

Keywords. Permutation Patterns, Möbius Function, Order Complex 


\section{Introduction}

Let $\sigma$ and $\pi$ be permutations of positive integers. We define an occurrence of $\sigma$ in $\pi$ to be a subsequence of $\pi$ with the same relative order of size as the letters in $\sigma$. For example, 132 occurs twice in 23541, as the subsequences 254 and 354 . The permutation poset $\mathcal{P}$ consists of all permutations with the partial order $\sigma \leq \pi$ if there is an occurrence of $\sigma$ in $\pi$. An interval $[\sigma, \pi]$ in $\mathcal{P}$ is the subposet $\{z \in \mathcal{P} \mid \sigma \leq z \leq \pi\}$. The Möbius function of a poset is defined recursively as: $\mu(a, b)=0$ if $a \not \leq b, \mu(a, a)=1$ for all $a$ and, for $a<b$ :

$$
\mu(a, b)=-\sum_{a \leq z<b} \mu(a, z) .
$$

In this paper we present a two term formula for the Möbius function of intervals $[\sigma, \pi]$ in $\mathcal{P}$. Its first term is, up to a sign, the number of certain occurrences of $\sigma$ in $\pi$, which can be computed in time polynomial in the length of $\pi$, whereas the second term requires exponential time to compute. However, this second term seems to be zero for a substantial proportion of intervals. This new formula thus seems to be the first instance of a polynomial time formula for the Möbius function of intervals in $\mathcal{P}$ that applies to a significant proportion of intervals.

The modern study of permutation patterns can be traced to Knuth's 1968 work [Knu68], where it was shown that permutations that avoid 231, that is, contain no occurrence of 231 , can be sorted using a stack. This link between sorting complexity and permutation avoidance was further investigated in the 1970s and 1980s in papers such as [Knu70], [Rog78], [Rot81] and [SS85]. In recent decades hundreds of papers have been published in the field of permutation patterns, an excellent review of the field's current state can be found in [Kit11] and many open problems can be found in [Ste13].

The study of the Möbius function of $\mathcal{P}$ was first proposed in [Wil02] and the first results appeared in [SV06], where a formula for intervals of layered permutations was presented. A layered permutation is the direct sum of decreasing permutations, where the direct sum $\sigma \oplus \pi$ of two permutations $\sigma$ and $\pi$ is obtained by appending $\pi$ to $\sigma$ after adding the length of $\sigma$ to each letter of $\pi$. For example, $312 \oplus 213=$ 312546. There is an analogous skew sum $\sigma \ominus \pi$, where $\pi$ is appended to $\sigma$ after the length of $\pi$ is added to each element of $\sigma$. In [BJJS11] a formula for the Möbius function is presented for intervals of decomposable permutations, that is, permutations that can be written as the direct sum of two or more non-empty permutations. However this formula is recursive and bottoms out in intervals bounded by indecomposable permutations, for which there is no general formula for the Möbius function.

Furthermore, in [BJJS11] a formula is presented for intervals of separable permutations, that is, permutations that avoid 2413 and 3142, or equivalently, permutations that can be written using only direct sums, skew sums and the singleton permutation 1. A formula for the Möbius function of intervals of permutations with a fixed number of descents is given in [Smi16], where a descent occurs at position $i$ in a permutation $\pi=\pi_{1} \ldots \pi_{n}$ if $\pi_{i}>\pi_{i+1}$. Further results have been presented in [ST10, Smi14, MS15]. However, the proportion of intervals $[\sigma, \pi]$ which satisfy any of these properties approaches zero as the length of $\pi$ increases. There are indications that the formula we present here reduces the computation of the Möbius function to polynomial time for a significant proportion of intervals.

Many of the results on the Möbius function of intervals of $\mathcal{P}$, and also of some posets of words, are linked to the number of what have been termed normal occurrences, or normal embeddings, in the literature, see [Bjö90, Bjö93, SV06, BJJS11, Smi16]. The first appearance of normal occurrences is in Björner's paper [Bjö90], where a formula for the Möbius function of intervals of words with subword order is presented. The definition of a normal occurrence has varied in these papers, but all follow a similar 
theme.

Our definition of normal occurrences, which is simpler than most previous ones, is based upon the adjacencies of a permutation, where an adjacency in a permutation is a maximal sequence of increasing or decreasing consecutively valued letters in consecutive positions and the tail of an adjacency is all but its first letter. A normal occurrence of $\sigma$ in $\pi$, in our definition, is any occurrence that includes all the tails of all the adjacencies of $\pi$.

We present a formula, in Theorem 21 that shows the Möbius function of $[\sigma, \pi]$ is, up to a sign, equal to the number of normal occurrences of $\sigma$ in $\pi$ plus an extra term that seems to vanish for a significant proportion of intervals. For example, we know this extra term vanishes if $\sigma$ and $\pi$ have the same number of descents, which is a consequence of the result in [Smi16]. Using interval blocks, which appear in [ST10], we prove that if for all permutations $\lambda \in[\sigma, \pi)$ there is a singleton interval block, that is, a letter of $\pi$ which belongs to no occurrence of $\lambda$, the second term of the formula vanishes. The above mentioned cases are of zero proportion when the length of $\pi$ goes to infinity, but computer tests indicate that for a substantial proportion of intervals the second term of our formula vanishes. Why that is the case is still a mystery, but this suggests that many more families of intervals than are now known may turn out to have a tractable Möbius function.

It is shown in [MS15] that if $\pi$ is decomposable and has equal consecutive components then for any subpermutation $\sigma$ obtained by removing $k>1$ of the equal components, the interval $[\sigma, \pi]$ contains a disconnected subinterval. Many of the definitions of normal occurrences have an extra condition for the case when $\pi$ has this property. We prove a result that indicates the second term of our formula for the Möbius function is often nonzero in this case. Exactly what the connection is between this second term and the topology of such intervals is another mystery.

Computing the Möbius function using the original recursive formula has exponential complexity, whereas our formula splits the computation into two parts. The first part, that is, computing the number of normal occurrences, can be done in polynomial time and the second part has exponential complexity in the general case. Our formula here is the first formula for arbitrary intervals of permutations that seems to have polynomial time complexity for a significant proportion of intervals.

In the following sections we present some of the results from the paper [Smi15], we refer the reader to the paper for any proofs which are omitted here. In Section 2 we introduce some definitions and give a brief introduction to the topology of posets. Furthermore, we present a poset $A^{\sigma, \pi}$ which is the union of certain posets defined by the occurrences of $\sigma$ in $\pi$. We present a surjective poset map from $A^{\sigma, \pi}$ onto $[\sigma, \pi]$ that we later use to compute $\mu(\sigma, \pi)$. In Section 3 we present and prove our main result, that the Möbius function of intervals of $\mathcal{P}$ equals the number of normal occurrences plus an extra term that we define. In Section 4 we apply our formula to show that the Möbius function of $[\sigma, \pi]$ equals the number of normal occurrences of $\sigma$ in $\pi$ if for each $\lambda \in[\sigma, \pi)$ there is at least one letter of $\pi$ which is not in any occurrence of $\lambda$. We also show that the value of the second term of our formula for the Möbius function of $[\sigma, \pi]$ is often nonzero when $\pi$ has a decomposition into a direct sum with consecutive equal components. Such repeated components have played a (different) role in work by other authors, but the connection remains mysterious.

\section{Definitions and Preliminaries}

In this section we introduce some definitions required to present our main result. Two nonequal permutations can have the same pattern but on different letters. We wish to treat such permutations as the same so 
we consider permutations in their smallest form, this is made formal by the following definition:

Definition 1 Given a permutation $\pi$ the reduced form of $\pi$ denoted $\operatorname{red}(\pi)$ is obtained by replacing the $i$-th smallest letter in $\pi$ with the letter $i$.

Example 2 The reduced form of $\pi=25473$ is $\operatorname{red}(\pi)=14352$.

We assume throughout that a permutation is in its reduced form, unless otherwise stated.

Now we introduce an important property of permutations that is fundamental to our results:

Definition 3 An adjacency in a permutation is a maximal sequence of increasing or decreasing consecutively valued letters in consecutive order. An adjacency with a single letter is a trivial adjacency. The tail of a nontrivial adjacency is all but the first letter of the adjacency and a trivial adjacency does not have a tail.

Example 4 The permutation $\pi=2314765$ has adjacencies 23, 1, 4 and 765 and the tails are 3 and 65 .

Next we define embeddings and our version of normal embeddings. Embeddings are in one-to-one correspondence with occurrences, and we use embeddings instead of occurrences throughout the rest of the paper because they allow for easier presentation of the required definitions.

Definition 5 Consider the permutations $\sigma \leq \pi$. An embedding $\eta$ of $\sigma$ in $\pi$ is a sequence of the same length as $\pi$ such that the nonzero letters in $\eta$ are the letters of an occurrence of $\sigma$ in $\pi$ and in the same positions in $\eta$ as in $\pi$.

An embedding $\eta$ of $\sigma$ in $\pi$ is normal if the positions of all the letters in all the tails of the adjacencies in $\pi$ are nonzero in $\eta$. We denote the number of normal embeddings of $\sigma$ in $\pi$ as $\mathrm{NE}(\sigma, \pi)$.

Example 6 For $\sigma=132$ and $\pi=2314765$ the sequence 0300065 is the only normal embedding of $\sigma$ in $\pi$, so $\mathrm{NE}(\sigma, \pi)=1$.

Proposition 7 Computing $\operatorname{NE}(\sigma, \pi)$ for a fixed $\sigma$ can be done in time polynomial in the length of $\pi$.

Proof: Counting the number of occurrences of $\sigma$ in $\pi$, of lengths $k$ and $n$, respectively, can be done in polynomial time $\mathcal{O}\left(n^{k}\right)$ by exhaustive search, and testing for normality is linear.

We use the adjacencies of a permutation to break down the permutation and embeddings into smaller components.

Definition 8 Consider permutations $\sigma \leq \pi$ and an embedding $\eta$ of $\sigma$ in $\pi$. Let $\hat{\pi}=\left(\hat{\pi}_{1}, \ldots, \hat{\pi}_{t}\right)$ be the decomposition of $\pi$ into its adjacencies, that is, $\hat{\pi}_{i}$ is the increasing or decreasing permutation corresponding to the $i$-th adjacency of $\pi$.

Define $\hat{\eta}:=\left(\hat{\eta}_{1}, \ldots, \hat{\eta}_{t}\right)$, where $\hat{\eta}_{i}$ is the permutation obtained from the nonzero letters that $\eta$ embeds in the $i$-th adjacency of $\pi$. If $\eta$ does not embed in any letters of the $i$-th adjacency then $\hat{\eta}_{i}=\emptyset$.

Example 9 If $\sigma=132$ and $\pi=2314765$ then $\hat{\pi}=(12,1,1,321)$ and the embedding $\eta=0010760$ gives $\hat{\eta}=(\emptyset, 1, \emptyset, 21)$.

When considering embeddings the selection of letters within an adjacency is usually irrelevant. This is made formal by the following equivalence relation. 
Definition 10 Let $E^{\sigma, \pi}$ be the set of embeddings of $\sigma$ in $\pi$. Define an equivalence relation on embeddings where $\eta \sim \psi$ if the only differences between $\eta$ and $\psi$ occur within adjacencies of $\pi$. Define $\widehat{E}^{\sigma, \pi}$ as the set containing the rightmost embedding, that is, the embedding where the nonzero letters are the furthest right, of each equivalence class of $E^{\sigma, \pi} / \sim$.

Consider $\eta \in \widehat{E}^{\sigma, \pi}$ and define the zero set of $\eta$ as $Z(\eta)=\left\{i \mid \eta_{i}=0\right\}$. Define $E Z^{\sigma, \pi}$ to be the set of sets of embeddings in $\widehat{E}^{\sigma, \pi}$ such that for each set $S \in E Z^{\sigma, \pi}$ we have $\bigcap_{\eta \in S} Z(\eta)=\emptyset$.

When defining $\widehat{E}^{\sigma, \pi}$ we choose the rightmost embedding to ensure that all normal embeddings are in $\widehat{E}^{\sigma, \pi}$. Note that if $\eta \sim \psi$ then $\hat{\eta}=\hat{\psi}$, which can be used as an equivalent definition of the equivalence relation. The set $E Z^{\sigma, \pi}$ is upwards closed under containment because if we take any set $S \in E Z^{\sigma, \pi}$ adding a new embedding to $S$ will result in a set that still has empty intersection of zero sets.

Example 11 If $\sigma=132$ and $\pi=413265$ then the embedding 013200 has zero set $Z(013200)=\{1,5,6\}$ and

$$
\begin{aligned}
E^{\sigma, \pi}= & \{013200,400065,010065,003065,000265\}, \\
\widehat{E}^{\sigma, \pi}= & \{013200,400065,010065,000265\}, \\
E Z^{\sigma, \pi}= & \{\{013200,400065\},\{013200,400065,010065\}, \\
& \{013200,400065,000265\},\{013200,400065,010065,000265\}\} .
\end{aligned}
$$

Using our decomposition we build posets from embeddings in the following way:

Definition 12 Given an embedding $\eta \in E^{\sigma, \pi}$ define the poset $P(\eta):=\left[\hat{\eta}_{1}, \hat{\pi}_{1}\right] \times \cdots \times\left[\hat{\eta}_{t}, \hat{\pi}_{t}\right]$ and

$$
A^{\sigma, \pi}:=\bigcup_{\eta \in \widehat{E}^{\sigma, \pi}} P(\eta)^{o},
$$

where $P(\eta)^{o}$ denotes the interior of $P(\eta)$, that is, $P(\eta)$ with the top and bottom elements removed.

Example 13 Consider $[132,413265]$ and let $\eta_{1}, \eta_{2}, \eta_{3}$ and $\eta_{4}$ be the embeddings listed in $\widehat{E}^{\sigma, \pi}$ in Example 11. Then $\hat{\pi}=(1,1,21,21)$ and $\hat{\eta}_{1}=(\emptyset, 1,21, \emptyset), \hat{\eta}_{2}=(1, \emptyset, \emptyset, 21), \hat{\eta}_{3}=(\emptyset, 1, \emptyset, 21)$ and $\hat{\eta}_{4}=(\emptyset, \emptyset, 1,21)$. See Figure 1 for $P\left(\eta_{i}\right)$ and $A^{132,413265}$.

The poset $A^{\sigma, \pi}$ consists of the elements $\hat{\eta}$ for all $\eta \in \widehat{E}^{\lambda, \pi}$ and all $\lambda \in(\sigma, \pi)$. Therefore, we define a surjective poset map $f$ from $A^{\sigma, \pi}$ to $(\sigma, \pi)$ in the following way:

Definition 14 Let $f: A^{\sigma, \pi} \rightarrow(\sigma, \pi)$ be the map which maps all elements $\hat{\eta}$, where $\eta \in \widehat{E}^{\lambda, \pi}$, to $\lambda$.

Example 15 If $[132,413265]$ and $\hat{\eta}=(1, \emptyset, 1,21)$ then $\eta=400265 \in \widehat{E}^{2143, \pi}$, so $f(\hat{\eta})=2143$.

\subsection{The Topology of a Poset}

We study the topology of a poset by constructing a simplicial complex from the poset in the following way:

Definition 16 Let $P$ be a poset. A chain in $P$ is a totally ordered subset $\left\{z_{1}<\cdots<z_{t}\right\}$. The order complex of $P$, denoted $\Delta(P)$, is the simplicial complex whose vertices are the elements of $P$ and whose faces are the chains of $P$. 

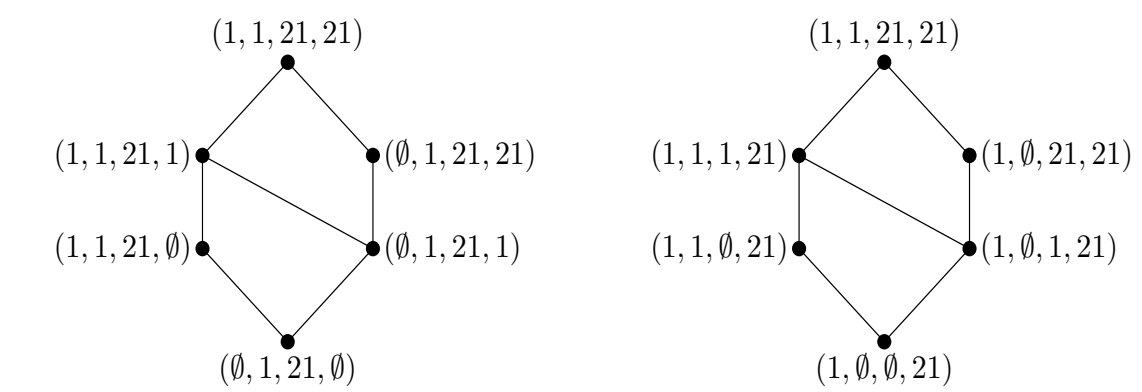

$P(013200)=[\emptyset, 1] \times[1,1] \times[21,21] \times[\emptyset, 21] \quad P(400065)=[1,1] \times[\emptyset, 1] \times[\emptyset, 21] \times[21,21]$
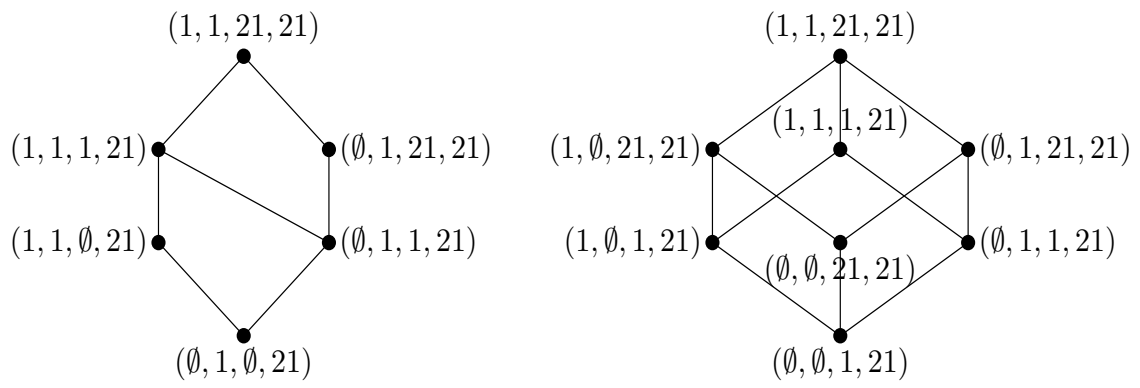

$P(010065)=[\emptyset, 1] \times[1,1] \times[\emptyset, 21] \times[21,21] \quad P(000265)=[\emptyset, 1] \times[\emptyset, 1] \times[1,21] \times[21,21]$

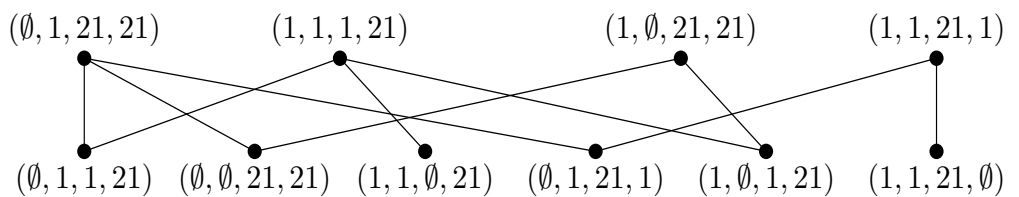

$A^{132,413265}$

Fig. 1: The posets of the embeddings of 132 in 413265 and the union $A^{132,413265}$ of their interiors. 
When we refer to the order complex of an interval $[\sigma, \pi]$ we mean the order complex of the interior $(\sigma, \pi)$, which we denote $\Delta(\sigma, \pi)$.

Example 17 Consider the interval $I=[123,4567123]$. An example of a chain in $(123,4567123)$ is $4123<456123$. The order complex and Hasse diagram of I are given in Figure 2

When we mention a topological property of a poset we refer to the corresponding property of its order complex. For further background on order complexes and poset topology in general see [Wac07].

We can use the order complex of $[\sigma, \pi]$ to calculate $\mu(\sigma, \pi)$ due to the following formula, which is an application of the Philip Hall Theorem and the Euler-Poincaré formula for the reduced Euler characteristic, see [Wac07, Section 1.2]:

$$
\mu(\sigma, \pi)=\tilde{\chi}(\Delta(\sigma, \pi))=\sum_{i=-1}^{|\pi|-|\sigma|}(-1)^{i} \tilde{\beta}_{i}(\Delta(\sigma, \pi)),
$$

where $\tilde{\chi}$ is the reduced Euler characteristic and $\tilde{\beta}_{i}$ is the $i$-th reduced Betti number, that is, the rank of the $i$-th reduced homology group. Therefore, by calculating the homology of $[\sigma, \pi]$ we can compute the Möbius function. For example, if we can show that $\Delta(\sigma, \pi)$ is contractible this implies $\mu(\sigma, \pi)=0$, and if $\Delta(\sigma, \pi)$ and $\Delta(\alpha, \beta)$ are homotopically equivalent then $\mu(\sigma, \pi)=\mu(\alpha, \beta)$.

The first explicit results on the topology of intervals of permutations appear in [MS15] and [Smi16].
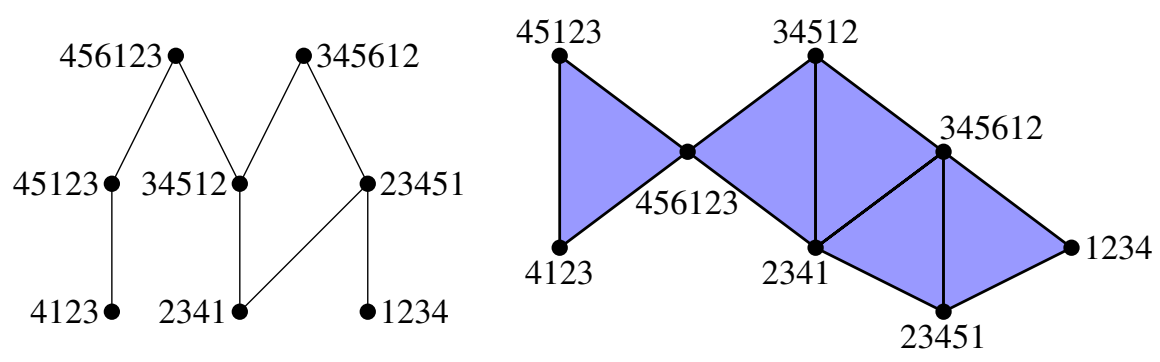

Fig. 2: Left: Hasse diagram of $(123,4567123)$. Right: The order complex $\Delta(123,4567123)$.

\section{The Main Result}

We use the map $f$ in Definition 14 to calculate the Möbius function of $[\sigma, \pi]$ by calculating $\mu\left(A^{\sigma, \pi}\right)$ and the effect on the Möbius function when applying $f$. First we compute $\mu\left(A^{\sigma, \pi}\right)$. Given a set $A$ of posets the Möbius function of the union of $A$ can be calculated using the following inclusion-exclusion formula, which can be seen as a consequence of the inclusion-exclusion formula for the Euler characteristic and Equation (1):

$$
\mu\left(\bigcup_{a \in A} a\right)=\sum_{\substack{S \subseteq A \\ S \neq \emptyset}}(-1)^{|S|-1} \mu\left(\bigcap_{a \in S} a\right)
$$


Applying Equation (2) to $A^{\sigma, \pi}$ gives:

$$
\mu\left(A^{\sigma, \pi}\right)=\sum_{\substack{S \subseteq \widehat{E}^{\sigma, \pi} \\ S \neq \emptyset}}(-1)^{|S|-1} \mu\left(\bigcap_{\eta \in S} P(\eta)^{o}\right) .
$$

To calculate this we need to know the Möbius function of the intersections $\cap_{\eta \in S} P(\eta)^{o}$. Note that when calculating the Möbius function of the interior (or intersection of interiors) we add the top and bottom elements back in. Therefore, a contractible intersection has Möbius function 0 , an empty intersection has Möbius function -1 and $\mu\left(P(\eta)^{\circ}\right)=\mu(P(\eta))$. See [Smi15] for the proof of Lemma 18 .

Lemma 18 If $S \subseteq \widehat{E}^{\sigma, \pi}$ and $|S|>1$ then:

$$
\mu\left(\bigcap_{\eta \in S} P(\eta)^{o}\right)=\left\{\begin{array}{ll}
-1, & \text { if } S \in E Z^{\sigma, \pi} \\
0, & \text { otherwise }
\end{array} .\right.
$$

Example 19 Consider our running example of $[132,413265]$. Let $S=\{013200,010065\}$ then $S \notin$ $E Z^{\sigma, \pi}$ because 1 is in the zero set of both embeddings. Therefore, Lemma 18 implies $\mu\left(P(013200)^{\circ} \cap\right.$ $\left.P(010065)^{\circ}\right)=0$. We can check this by looking at Figure 1 where we can see that the intersection $P(013200) \cap P(010065)$ is the single point $(\emptyset, 1,21,21)$, thus the Möbius function equals zero.

Now that we know the Möbius function of the intersections we can compute $\mu\left(A^{\sigma, \pi}\right)$ :

Lemma 20

$$
\mu\left(A^{\sigma, \pi}\right)=(-1)^{|\pi|-|\sigma|} \mathrm{NE}(\sigma, \pi)+\sum_{S \in E Z^{\sigma, \pi}}(-1)^{|S|} .
$$

Proof: We can split Equation (3) into two parts:

$$
\mu\left(A^{\sigma, \pi}\right)=\sum_{\eta \in \widehat{E}^{\sigma, \pi}} \mu\left(P(\eta)^{o}\right)+\sum_{\substack{S \subseteq \widehat{E}^{\sigma, \pi} \\|S|>1}}(-1)^{|S|-1} \mu\left(\bigcap_{\eta \in S} P(\eta)^{o}\right) .
$$

By Lemma 18 the second part of the right hand side of Equation (4) equals $\sum_{S \in E Z^{\sigma, \pi}}(-1)^{|S|}$.

By the definition of $P(\eta)$, and the identity $\mu(A \times B)=\mu(A) \mu(B)$, we know $\mu(P(\eta))=\prod_{1 \leq i \leq t} \mu\left(\hat{\eta}_{i}, \hat{\pi}_{i}\right)$.

We know that $\left[\hat{\eta}_{i}, \hat{\pi}_{i}\right]$ is always a chain, so by the definition of normality if $\eta$ is not normal there is some $i$ such that $\left|\hat{\eta}_{i}\right| \leq\left|\hat{\pi}_{i}\right|-2$, so $\mu\left(\hat{\eta}_{i}, \hat{\pi}_{i}\right)=0$ which implies $\mu(P(\eta))=0$. If $\eta$ is normal then $\left|\hat{\pi}_{i}\right|-\left|\hat{\eta}_{i}\right|=0$ or 1 , so $\mu\left(\hat{\eta}_{i}, \hat{\pi}_{i}\right)=1$ or -1 , for all $i$. There are $|\pi|-|\sigma|$ parts $\left[\hat{\eta}_{i}, \hat{\pi}_{i}\right]$ with $\mu\left(\hat{\eta}_{i}, \hat{\pi}_{i}\right)=-1$, one for each zero in $\eta$, and the remaining have $\mu\left(\hat{\eta}_{i}, \hat{\pi}_{i}\right)=1$. Therefore, $\mu\left(P(\eta)^{o}\right)=\mu(P(\eta))=(-1)^{|\pi|-|\sigma|}$ for each normal embedding, so the first term in the right hand side of Equation (4) equals $(-1)^{|\pi|-|\sigma|} \mathrm{NE}(\sigma, \pi)$.

We now present our formula for the Möbius function that applies to any interval of permutations: 
Theorem 21 For any permutations $\sigma$ and $\pi$ :

$$
\mu(\sigma, \pi)=(-1)^{|\pi|-|\sigma|} \mathrm{NE}(\sigma, \pi)+\sum_{\lambda \in[\sigma, \pi)} \mu(\sigma, \lambda) \sum_{S \in E Z^{\lambda, \pi}}(-1)^{|S|} .
$$

Proof: The poset $(\sigma, \pi)$ is obtained from $A^{\sigma, \pi}$ after taking a quotient along the fibers of the map $f$. So for each $\lambda \in(\sigma, \pi)$ the set $\widehat{E}^{\lambda, \pi}$ is mapped a point we denote $\lambda$. This transforms $A^{\sigma, \pi}$ into the interval $(\sigma, \pi)$. We need to know what effect this has on the Möbius function of $A^{\sigma, \pi}$.

We work our way from the bottom to the top, so we can assume that all elements below the elements of $\widehat{E}^{\lambda, \pi}$ have already been quotiented and all elements above have not. Define the poset $W(\lambda):=\{\tau \in$ $A^{\sigma, \pi} \mid \tau \leq \eta$ or $\tau \geq \eta$ for some $\left.\eta \in \widehat{E}^{\lambda, \pi}\right\}$. When we quotient the elements of $\widehat{E}^{\lambda, \pi}$ to $\lambda$ we map $W(\lambda)$ onto a contractible poset, since in that poset the element $\lambda$ is comparable to all other elements and thus represents a cone point in the corresponding order complex. This implies the change to the Möbius function is $-\mu(W(\lambda))$.

To compute $\mu(W(\lambda))$ we split $W(\lambda)$ into two disjoint parts

$$
\begin{aligned}
& W(\lambda)^{<}:=\left\{\tau \in W(\lambda) \mid \tau<\eta \text { for some } \eta \in \widehat{E}^{\lambda, \pi}\right\}, \\
& W(\lambda)^{\geq}:=\left\{\tau \in W(\lambda) \mid \tau \geq \eta \text { for some } \eta \in \widehat{E}^{\lambda, \pi}\right\} .
\end{aligned}
$$

The poset $W(\lambda)<$ is isomorphic to $(\sigma, \lambda)$ because all points below $\lambda$ have already been quotiented. The poset $W(\lambda) \geq$ is equal to $\bigcup_{\eta \in \widehat{E}^{\lambda, \pi}}(P(\eta) \backslash \hat{\pi})$ which has Möbius function $-\sum_{S \in E Z^{\lambda, \pi}}(-1)^{|S|}$, by Lemma 18 and the inclusion-exclusion formula (this also follows from the Crosscut Theorem, see [Sta12. Corollary 3.9.4]).

Because every element of $\widehat{E}^{\lambda, \pi}$ lies above every element of $(\sigma, \lambda)$ this implies $W(\lambda)=W(\lambda)^{<} \star$ $W(\lambda) \geq$, where $\star$ denotes the topological join. Therefore,

$$
-\mu(W(\lambda))=-\mu\left(W(\lambda)^{<}\right) \mu\left(W(\lambda)^{\geq}\right)=\mu(\sigma, \lambda) \sum_{S \in E Z^{\lambda, \pi}}(-1)^{|S|} .
$$

So we start with $\mu\left(A^{\sigma, \pi}\right)$, given by Lemma 20, and then subtract $\mu(W(\lambda))$ for each $\lambda \in(\sigma, \pi)$, which gives the desired formula.

Remark 22 Computer tests indicate that $95 \%$ of intervals $[\sigma, \pi]$, where $|\pi|<9$, satisfy $\mu(\sigma, \pi)=$ $(-1)^{|\pi|-|\sigma|} \mathrm{NE}(\sigma, \pi)$. Thus, for these intervals the latter term in Equation (5) is zero.

Remark 23 The complexity of counting the number of normal embeddings is polynomial so in the cases where we can show that the latter term of Equation [5] equals zero we have a polynomial time formula for the Möbius function. This is a dramatic improvement over the original recursive formula that has exponential complexity. However, computing the latter term of Equation (5) also has exponential complexity as it requires us to compute the entire interval $[\sigma, \pi]$.

Tests show that using Equation (5) is often much quicker than computing the Möbius function using the recursive formula. When computing the Möbius function of the rank 15 interval

[54123, 9710481265319172014181112161513$]$ 
the formula in Equation (5) took 1.75 minutes and the recursive formula took 13.5 hours. Note that this interval has Möbius function -3 but no normal embeddings so the latter term of Equation (5) is nonzero in this case. Furthermore, using Equation (5) we were able to compute the Möbius function of a rank 16 interval in 1 hour and a rank 17 interval in 6 hours. However, if $\sigma$ has a large number of occurrences in $\pi$ then using Equation (5) can be quite slow. For example, if $\sigma=2413$ and $\pi=24681013579$ then there are 35 occurrences of $\sigma$ in $\pi$ and $\mu(\sigma, \pi)$ can be computed in 0.06 seconds using the recursive formula but takes 15.5 hours using Equation (5).

\section{Applications}

Due to Remark 22 we suspect that the second part of Equation (5) vanishes for a significant proportion of intervals. For example, a consequence of Proposition 3.3 in [Smi16] is that if $\sigma$ and $\pi$ have the same number of descents then the second part of Equation (5) vanishes. The key to simplifying Equation (5) is answering the following question:

Question 24 Given an interval $[\sigma, \pi]$, for which $\lambda \in[\sigma, \pi)$ is the following sum nonzero?:

$$
\mathrm{EZ}(\lambda, \pi):=\sum_{S \in E Z^{\lambda, \pi}}(-1)^{|S|} .
$$

One case where $\operatorname{EZ}(\lambda, \pi)=0$ is when the set $E Z^{\lambda, \pi}$ is always empty, which leads us to the following definition and proposition:

Definition 25 We say an interval $[\sigma, \pi]$ has a single block if there exists some $i$ such that $\eta_{i}=0$ for any $\eta \in \widehat{E}^{\sigma, \pi}$. That is, there is a letter in $\pi$ that is not contained in any of the occurrences in $\widehat{E}^{\sigma, \pi}$.

We say an interval is single if for all $\lambda \in[\sigma, \pi)$ the interval $[\lambda, \pi]$ has a single block.

Our notation here follows from the idea of interval blocks in [ST10]. Computer tests show that $78.6 \%$ of intervals $[\sigma, \pi]$, where $|\pi|<9$, are single and we found that $39 \%$ of 48300 random rank 10 intervals, where $|\sigma|=5$ and $|\pi|=15$, are single. We suspect that the proportion of intervals that are single approaches zero as the rank tends to infinity, because the likelihood that there exists some $\lambda \in[\sigma, \pi]$ such that $[\lambda, \pi]$ does not have a single block increases as the rank of the interval increases.

Proposition 26 If $[\sigma, \pi]$ is single then $\mu(\sigma, \pi)=(-1)^{|\pi|-|\sigma|} \mathrm{NE}(\sigma, \pi)$.

Intervals that contain a disconnected subinterval of rank at least 3 are non-shellable, as shown by Björner in [Bjö80], and thus not amenable to some of the elegant methods of topological combinatorics, see [MS15] for further background. To finish we consider a particular type of interval $[\sigma, \pi]$ that is known to be disconnected and show that $\operatorname{EZ}(\sigma, \pi) \neq 0$ for these intervals. Whether there is a topological "reason" for $\operatorname{EZ}(\sigma, \pi)$ being nonzero in these cases we do not know.

We consider decomposable permutations and write them in the form $\pi_{1} \oplus \cdots \oplus \pi_{n}$, where each $\pi_{i}$, which we call a component of $\pi$, is indecomposable. Consider a permutation $\pi$, with a sequence of $\alpha \geq 2$ equal consecutive components, and $\lambda \leq \pi$ obtained from $\pi$ by removing $k$ of the components from this sequence, where $1 \leq k<\alpha$. The interval $[\lambda, \pi]$ is disconnected, which follows from results in [MS15], specifically Lemma 4.2 and Theorem 5.6. These intervals are the cause of the extra conditions in the formulas for the Möbius function that appear in [BJJS11] and [MS15].

We compute the extra term for these cases with the following proposition: 
Proposition 27 Consider a decomposable permutation $\pi=\pi_{1} \oplus \cdots \oplus \pi_{n}$ with a sequence of consecutive components $\pi_{i+1}=\cdots=\pi_{i+\alpha}$, with $\alpha>1$. Let $\lambda=\lambda_{1} \oplus \cdots \oplus \lambda_{m}$ be the subpermutation of $\pi$ obtained by reducing the sequence of equal components to length $\ell$, for $0 \leq \ell \leq \alpha$. Then:

$$
\mathrm{EZ}(\lambda, \pi)=(-1)^{\alpha-\ell-1}\left(\begin{array}{c}
\alpha-1 \\
\ell-1
\end{array}\right) .
$$

See [Smi15] for the proof of Proposition 27] and see Example 29 for an application of Proposition 27 We conjecture that we can generalise this further by removing elements from more than one sequence of equal components:

Conjecture 28 Consider a decomposable permutation $\pi=\pi_{1} \oplus \cdots \oplus \pi_{n}$ which has $t$ sequences of equal components $\pi_{i+1}=\cdots=\pi_{i+\alpha_{i}} \neq 1$ of respective lengths $\alpha_{i}$, for $1 \leq i \leq t$. Let $\lambda=\lambda_{1} \oplus \cdots \oplus \lambda_{m}$ be the permutation obtained from $\pi$ by, for each $i$, reducing the $i$-th sequence to length $\ell_{i}$, with $0 \leq \ell_{i} \leq \alpha_{i}$. Then:

$$
\operatorname{EZ}(\lambda, \pi)=(-1)^{\alpha-\ell-1} \prod_{i=1}^{t}\left(\begin{array}{c}
\alpha_{i}-1 \\
\ell_{i}-1
\end{array}\right)
$$

where $\alpha=\alpha_{1}+\cdots+\alpha_{t}$ and $\ell=\ell_{1}+\cdots+\ell_{t}$.

Example 29 Consider the permutation $\pi=21437561089131112$ which has the decomposition $21 \oplus$ $21 \oplus 312 \oplus 312 \oplus 312$, so has 2 sequences of equal components.

First consider $\tau=21 \oplus 21 \oplus 321 \oplus 321$. We have decreased the length $\alpha=3$ sequence of 312 's to length $\ell=2$. Applying Proposition 27 gives:

$$
\mathrm{EZ}(\tau, \pi)=(-1)^{3-2-1}\left(\begin{array}{l}
3-1 \\
2-1
\end{array}\right)=2 .
$$

Now consider $\lambda=21 \oplus 312 \oplus 312=21534867$, which has $\mathrm{EZ}(\lambda, \pi)=-2$. We see that Conjecture 28 gives us the same value, where $\alpha_{1}=2, \ell_{1}=1$ and $\alpha_{2}=3, \ell_{2}=2$ :

$$
(-1)^{5-3-1}\left(\begin{array}{l}
2-1 \\
1-1
\end{array}\right)\left(\begin{array}{l}
3-1 \\
2-1
\end{array}\right)=-2 .
$$

\section{References}

[BJJS11] Alexander Burstein, Vít Jelínek, Eva Jelínková, and Einar Steingrímsson. The Möbius function of separable and decomposable permutations. Journal of Combinatorial Theory. Series A, 118(8):2346-2364, 2011.

[Bjö80] Anders Björner. Shellable and Cohen-Macaulay partially ordered sets. Transactions of the American Mathematical Society, 260(1):159-183, 1980.

[Bjö90] Anders Björner. The Möbius function of subword order. Institute for Mathematics and its Applications, 19:118, 1990.

[Bjö93] Anders Björner. The Möbius function of factor order. Theoretical Computer Science, 117(1):91-98, 1993. 
[Kit11] Sergey Kitaev. Patterns in Permutations and Words. Monographs in Theoretical Computer Science. An EATCS Series. Springer, Heidelberg, 2011.

[Knu68] Donald E. Knuth. The Art of Computer Programming, Vol. 1: Fundamental Algorithms. Addison-Wesley, Reading, Mass, 1968.

[Knu70] Donald E. Knuth. Permutations, matrices, and generalized young tableaux. Pacific Journal of Mathematics, 34(3):709-727, 1970.

[MS15] Peter R. W. McNamara and Einar Steingrímsson. On the topology of the permutation pattern poset. Journal of Combinatorial Theory, Series A, 134:1-35, 2015.

[Rog78] D. G. Rogers. Ascending sequences in permutations. Discrete Mathematics, 22(1):35-40, 1978.

[Rot81] Doron Rotem. Stack sortable permutations. Discrete Mathematics, 33(2):185-196, 1981.

[Smi14] Jason P. Smith. On the Möbius function of permutations with one descent. The Electronic Journal of Combinatorics, 21:2.11, 2014.

[Smi15] Jason P. Smith. A formula for the Möbius function of the permutation poset based on a topological decomposition. arXiv preprint arXiv:1506.04406, 2015.

[Smi16] Jason P. Smith. Intervals of permutations with a fixed number of descents are shellable. Discrete Mathematics, 339(1):118 - 126, 2016.

[SS85] Rodica Simion and Frank W. Schmidt. Restricted permutations. European Journal of Combinatorics, 6(4):383 - 406, 1985.

[ST10] Einar Steingrímsson and Bridget Eileen Tenner. The Möbius function of the permutation pattern poset. Journal of Combinatorics, 1(1):39-52, 2010.

[Sta12] Richard P. Stanley. Enumerative Combinatorics. Vol. 1. Cambridge Studies in Advanced Mathematics. Cambridge University Press, second edition, 2012.

[Ste13] Einar Steingrímsson. Some open problems on permutation patterns. In Surveys in Combinatorics, 409, pages 239-263. London Mathematical Society Lecture Note Series, 2013.

[SV06] Bruce E. Sagan and Vincent Vatter. The Möbius function of a composition poset. Journal of Algebraic Combinatorics, 24(2):117-136, 2006.

[Wac07] Michelle L. Wachs. Poset topology: Tools and applications. In Geometric Combinatorics, volume 13 of IAS/Park City Math. Ser., pages 497-615. Amer. Math. Soc., 2007.

[Wil02] Herbert S. Wilf. The patterns of permutations. Discrete Mathematics, 257(2-3):575-583, 2002. Kleitman and combinatorics: a celebration (Cambridge, MA, 1999). 